\title{
Quantum hierarchic models for information processing
}

\author{
M.V.Altaisky \\ Joint Institute for Nuclear Research, Joliot Curie 6, Dubna, 141980, Russia; \\ and Space Research Institute RAS, Profsoyuznaya 84/32, Moscow, 117997, Russia \\ e-mail: altaisky@mx.iki.rssi.ru \\ N.E.Kaputkina \\ National University of Science and Technology "MISiS", \\ Leninsky prospect 4, Moscow, 119049, Russia \\ e-mail: nataly@misis.ru
}

Sep 12, 2011

\begin{abstract}
Both classical and quantum computations operate with the registers of bits. At nanometer scale the quantum fluctuations at the position of a given bit, say, a quantum dot, not only lead to the decoherence of quantum state of this bit, but also affect the quantum states of the neighboring bits, and therefore affect the state of the whole register. That is why the requirement of reliable separate access to each bit poses the limit on miniaturization, i.e, constrains the memory capacity and the speed of computation. In the present paper we suggest an algorithmic way to tackle the problem of constructing reliable and compact registers of quantum bits. We suggest to access the states of quantum register hierarchically, descending from the state of the whole register to the states of its parts. Our method is similar to quantum wavelet transform, and can be applied to information compression, quantum memory, quantum computations.
\end{abstract}

\section{Introduction}

Classical information can be always encoded in a sequence of bits, the entities with two classically distinguishable states. The miniaturization of the information processing units to nanometer scales imposes constraints on memory capacity and the speed of computation, whatever the algorithm is classical or quantum. This constraints arise from the Heisenberg uncertainty principle and from the openness of any computational system. The mean momentum transfer required to access an element of the size $\Delta x \sim 10^{1} \mathrm{~nm}$ exceeds $\frac{\hbar}{2 \Delta x}$. This 
corresponds to the electron velocity $v \sim 10^{5} \mathrm{~m} / \mathrm{s}$ and the energy of meV order. The interaction of the same element with the environment at room temperature $T \sim 300 \mathrm{~K}$ results in energy transfer $k_{B} T \sim 25 \mathrm{meV}$. The decrease of the operating voltage to less than one Volt makes nanoscale logical devices to operate with a few electrons only. Thus each logical state is achieved not with certainty, but with a finite probability, constrained by quantum effects.

Devoid of the environment a state of quantum bit is linear superposition of two basic states, 0 and 1 :

$$
|\psi\rangle=\alpha|0\rangle+\beta|1\rangle
$$

subjected to a unitary evolution $|\psi(t)\rangle=e^{-\imath t H}|\psi(0)\rangle$. The interaction with environment decoheres the state $|\psi\rangle$ into a classical mixture of the two basic states. When the interaction with the environment can be neglected the computation can be performed dissipation free by unitary evolution of quantum register in a parallel way according to quantum algorithms [1, 2]. Therefore there exists an obstacle that still prevents practical implementation of a workable quantum computer with more than a few quantum bits. This obstacle is the quantum decoherence - the loss of quantum information by means of relative dephasing of the qubits in the superposition of quantum states due to the interaction with environment.

The perspective candidates for memory qubits, as well as for quantum gates, are quantum dots - the artificial atoms of $10^{1} \mathrm{~nm}$ size with the spin of the excess electrons used as quantum bits [3, 4]. The spin of the excess electron in an isolated quantum dot can be reliably initialized to a ground state by optical pumping, or by thermal equilibration in a strong magnetic field. Having the relaxation time of $10^{-3}$ sec order [5], the electron spin of a quantum dot, having the spin decoherence time of $10^{-6}$ sec order [6], can be used as a qubit in quantum computations. Challenging problem is to control spin states of the electrons in an array of quantum dots, separated from each other by less than a typical optical wavelength size, rather than an electron spin in a single quantum dot [4].

The idea of the present paper is to arrange the quantum bits of a register hierarchically in blocks and process the blocks separately at each hierarchy level. In the next sections we will develop the necessary formalism to describe the Hilbert space of hierarchic states. We also present the physical models for hierarchic quantum registers based on the arrays of quantum dots.

The remainder of this paper is organized as follows. In Section 2 we generalize the ideas of the Mallat multiresolution analysis to quantum register. In Section 3 we discuss the CNOT quantum gate in a multiplet basis of quantum register. In Section 4 we discuss physical implementation of quantum gates on spin qubits based on quantum dots. In Conclusion we summarize the advantages of the quantum hierarchic information coding and the difficulties of manipulations with such registers. 


\section{Generalization of wavelet transform for quantum reg- isters}

Suppose we need to compress a large data vector $(N \gg 1)$ in such a way that a few coefficients store the most significant information - perhaps, that distinguishes the given object from all others, - the next coefficients store some less significant details, etc. Such techniques, first proposed by Burt and Adelson for digital image coding [7], is known as pyramidal image compression algorithm. It is based on the idea, that each four pixels of an image can be considered as a block, so that only one value is required to quantify the "mean color" of the block, and three more values required to quantify the deviations of pixel colors from the block mean. The same procedure can be applied to the group of 4 blocks of $2 \times 2$ pixels each into 16 pixel block, and so fourth. The averaging operator is usually denoted by $H$, and is referred to as low-pass filter, the projection operator onto the space of averaging-lost details is denoted by $G$ and is referred to as high-pass filter. If no information is lost during compression, the low- and high-pass filters obey the condition $G^{*} G+H^{*} H=1$. $H$ and $G$ operators project the sequence of length $N$ onto the sequences of length $N / 2$ (so, that the total amount of information is conserved), decreasing the resolution twice at each step. For the one-dimensional data $s \in l^{2}(\mathbb{Z})$, the action of $H$ and $G$ filters can be written as

$$
(H s)_{i}=\sum_{n} h_{n-2 i} s_{n}, \quad(G s)_{i}=\sum_{n} g_{n-2 i} s_{n}
$$

The decomposition of the data vector with the $H$ and $G$ operators (1) according to the scheme

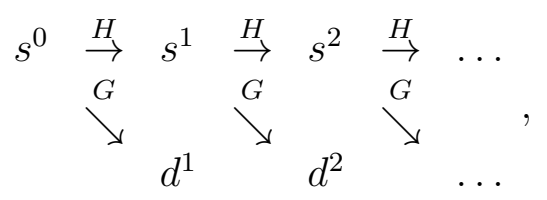

and appropriate reconstruction is known as fast wavelet transform algorithm [8].

The pyramidal image coding was generalized into the Mallat multiresolution analysis (MRA) [9]. The multiresolution analysis in $L^{2}(\mathbb{R})$, or the Mallat sequence, is an increasing sequence of closed subspaces $\left\{V_{j}\right\}_{j \in \mathbb{Z}}, V_{j} \in L^{2}(\mathbb{R})$, such that

1. $\ldots \subset V_{2} \subset V_{1} \subset V_{0} \subset V_{-1} \subset \ldots$

2. $\operatorname{clos} \cup_{j \in \mathbb{Z}} V_{j}=L^{2}(\mathbb{R})$

3. $\cap_{j \in \mathbb{Z}} V_{j}=\emptyset$

4. The spaces $V_{j}$ and $V_{j-1}$ are "similar": $f(x) \in V_{j} \Leftrightarrow f(2 x) \in V_{j-1}, \quad j \in \mathbb{Z}$ 
To set a basis on the Mallat sequence one needs to choose a scaling function $\phi(x)$, so that

$$
V_{j}=\text { linear } \operatorname{span}\left\{\phi_{k}^{j} ; j, k \in \mathbb{Z}\right\},
$$

where $\phi_{k}^{0}(x) \equiv \phi(x-k)$, and $\phi_{k}^{j}(x)=2^{-j / 2} \phi\left(2^{-j} x-k\right)$. Any function $f \in V_{1}$, due to the inclusion property 1 , can be written as a linear combination of the basic functions of $V_{0}$. Since the spaces $V_{j}$ and $V_{j+1}$ are different in resolution, some details are being lost when one sequentially projects a function $f \in V_{0}$ on a ladder of spaces $V_{1}, V_{2}, \ldots$. This details can be stored in the orthogonal complements $W_{j}=V_{j-1} \backslash V_{j}$. Explicitly: $V_{0}=W_{1} \oplus W_{2} \oplus W_{3} \oplus \ldots \oplus V_{M}$.

In quantum case we might also suggest a block structure of application of a linear operator to a quantum register. However we have first to consider a simpler question: How the qubits can be stored in quantum register, and can there exist any quantum structure similar to the Mallat sequence? The direct analogs of the Haar wavelet transform, based on quantum networks, have been already suggested for quantum computations [10, 11], but they implement a separate access to each quantum bit using the quantum gates, and can hardly form an effective memory.

Suppose we have a quantum register consisting of $N=2^{M}$ qubits and we are going to store $N^{\prime}<N$ quantum bits of information in it. The higher is the ratio $N / N^{\prime}$, the higher fidelity of information storage can be achieved: for more than one qubit can be used to store the same information. Such devices may be of practical use if depending on real amount of information to be stored different number of quantum bits is allocated.

Let us consider a quantum register implemented on spin-half particles:

$$
\otimes_{i=1}^{N}\left|s_{i}^{0}\right\rangle=\otimes_{i=1}^{N}\left(a_{i}^{0}|\uparrow\rangle_{i}^{0}+b_{i}^{0}|\downarrow\rangle_{i}^{0}\right), \quad\left|a_{i}^{0}\right|^{2}+\left|b_{i}^{0}\right|^{2}=1 .
$$

In contrast to classical bits the qubits take their values in the $S U(2)$ group, rather than in $\mathbb{R}$. So, the direct application of the Haar wavelet algorithm

$$
s_{k}^{j}=\frac{s_{2 k}^{j-1}+s_{2 k+1}^{j-1}}{\sqrt{2}}, \quad d_{k}^{j}=\frac{s_{2 k}^{j-1}-s_{2 k+1}^{j-1}}{\sqrt{2}}, \quad k=0, \ldots, 2^{M-j}-1,
$$

is not possible. However the spin state of a pair of fermions with spin $\frac{1}{2}$, considered as a compound boson, is completely determined by the product $\left|s_{a}\right\rangle \otimes\left|s_{b}\right\rangle$ by means of the Clebsch-Gordan coefficients; and vice versa: orthogonality of the Clebsch-Gordan coefficients allows to reconstruct the state of the pair of fermions from the known state of the boson they comprise [12].

If a product of $N$ fermion wave functions is decomposed into a direct sum of irreducible representations $T^{(J)}$, corresponding to the rotations of composite system:

$$
\otimes_{j} D^{(j)}=\oplus_{J} c_{J} T^{(J)},
$$

the compound system may be in either of the states $T^{(J)}$, for which the coefficient $c_{J}$ is not equal to zero. If a compound system was measured to be in a state $T^{(m)}$, then only those 
product terms $\left|s^{0}\right\rangle \otimes \cdots \otimes\left|s^{0}\right\rangle$ can survive, which contribute to the $T^{(m)}$ term in (3). The process of multiplication of the representations of angular momentum group can be done hierarchically, starting from pairs. The whole composite system can be described in terms of hierarchic state vectors

$$
\Phi=\left\{\left|s^{M}\right\rangle,\left|s^{M} s^{M-1}\right\rangle, \ldots,\left|s^{M} s_{M-1} \ldots s^{0}\right\rangle\right\} .
$$

In Hilbert space of hierarchic state vectors the reduced density matrix can be constructed by taking the trace over the states of the next hierarchy level [13]. Hierarchic representation provides an extra possibility to construct quantum gates by acting onto the states of the lower hierarchy level $(\alpha-1)$ depending on the states of the next hierarchic level $(\alpha)$ :

$$
\hat{B}=\left|\boldsymbol{i}^{(\alpha-1)}\right\rangle\left|\theta_{m}^{(\alpha)}\right\rangle B_{\boldsymbol{i k}}^{m}\left\langle\theta_{m}^{(\alpha)}\right|\left\langle\boldsymbol{k}^{(\alpha-1)}\right| .
$$

See [13] for details.

A sequence $\left\{s_{j}^{0}\right\}_{j}$ of bits can be hierarchically compressed using the projections onto the ladder of the Mallat sequence $V_{0}, V_{1}, \ldots, V_{M}$, where only the projections onto the orthogonal complements $W_{k}=V_{k-1} \backslash V_{k}$ can be kept in the memory. For the sequence of $2^{M}$ quantum bits the information can be encoded in the spin state of the whole system $\Psi=\prod_{k=0}^{2^{M}-1} \psi_{k}$, where $\psi_{k}$ is a two-component spinor describing the $k$-th qubit.

Let us consider simplest cases of $M=1$ and $M=2$.

For $\boldsymbol{M}=\mathbf{1}$ we have a pair of quantum bits. The composite wave function of such system transforms according to the representation

$$
D_{\frac{1}{2}} \otimes D_{\frac{1}{2}}=D_{1} \oplus D_{0}
$$

i.e. the total system can be either in triplet $\left(D_{1}\right)$ or in singlet $\left(D_{0}\right)$ state, or in their superposition. The bases of the product states (l.h.s. of (41)) and the composite system states (r.h.s. of (4)) are related by the linear transform (17). If the basis in two-qubit space is chosen as

$$
\left(e_{1}, e_{2}, e_{3}, e_{4}\right)=(|\downarrow\rangle|\downarrow\rangle,|\downarrow\rangle|\uparrow\rangle,|\uparrow\rangle|\downarrow\rangle,|\uparrow\rangle|\uparrow\rangle),
$$

and the basis in the space of states of composite boson is chosen as

$$
\left(s_{1}, s_{2}, s_{3}, s_{4}\right)=(|0,0\rangle,|1,-1\rangle,|1,0\rangle,|1,+1\rangle),
$$

they are related by a linear transform

$$
e_{i}=A_{i k} s_{k}, \quad A=\left(\begin{array}{cccc}
0 & 1 & 0 & 0 \\
-\frac{1}{\sqrt{2}} & 0 & \frac{1}{\sqrt{2}} & 0 \\
\frac{1}{\sqrt{2}} & 0 & \frac{1}{\sqrt{2}} & 0 \\
0 & 0 & 0 & 1
\end{array}\right) \text {. }
$$


For this system we can define the spaces $V_{0}, V_{1}, W_{1}$ as follows: $V_{0}$ is the product space transforming according to $D_{\frac{1}{2}} \otimes D_{\frac{1}{2}} ; V_{1}$ is the triplet state of the composite system, which transforms according to $D_{1}$ representation; $W_{1}$ is the singlet state of the compound system.

For $\boldsymbol{M}=\mathbf{2}$ the finest resolution space $V_{0}$ is the span of the four spinor product

$$
\Psi=\psi_{0} \psi_{1} \psi_{2} \psi_{3}
$$

which transforms according to $\left(D_{\frac{1}{2}} \otimes D_{\frac{1}{2}}\right) \otimes\left(D_{\frac{1}{2}} \otimes D_{\frac{1}{2}}\right)$.

We define $V_{1}$ as a linear span of the states of maximal spin of each block

$$
V_{1}=D_{1} \otimes D_{1}=D_{2} \oplus D_{1} \oplus D_{0} .
$$

In this case the detail space $W_{1}$ is

$$
W_{1}=V_{0} \backslash V_{1}=D_{1} \otimes D_{0}+D_{0} \otimes D_{1}+D_{0} \otimes D_{0}
$$

Similarly, the $V_{2}$ space is the maximal spin state of a next level block, which transforms according to $D_{2}$. The corresponding detailed space is

$$
W_{2}=V_{1} \backslash V_{2}=D_{1} \oplus D_{0}
$$

The total number of degrees of freedom is conserved. $V_{0}=W_{1} \oplus W_{2} \oplus V_{2}$. Their dimensions are $16=7+4+5$.

The information encoding in descending order saves the number of operations required to set the necessary configuration in $V_{0}$ space. For instance, in sufficiently strong magnetic field, the state $|2,2\rangle \in V_{2}$ of the whole system uniquely determines configuration of all 4 qubits. The decrease of magnetic field results in evolution of qubit pairs into singlet states.

\section{Gate operations}

The memory on spin states can be exploited in both classical [14, 15] and quantum [16] memory devices. Classical devices working on Boolean logic with AND and OR operations dissipate an energy of at least $k_{B} T \ln 2$ per logical step. The dissipation imposes a constraint on computation speed. Quantum computations are time-reversal, they do not dissipate the energy until the read-out of final result.

Quantum computation is performed by a sequence of unitary operations applied to the quantum register. Any conceivable unitary operation in quantum computing can be performed by sequential application of single-qubit gates and the two-qubit CNOT gate [17]. That is why the CNOT quantum gate is a key element of quantum information processing. The action of CNOT gate consists in the change of the second qubit state, if the first qubit, used as a control, is in the state "1". The action of the CNOT gate is defined by the rules, listed in Table 1 . 


$\begin{array}{cccr}\text { control } & \text { target } & \text { resulting } & S_{z} \\ \text { qubit } & \text { qubit } & \text { state } & \\ |\downarrow\rangle & |\downarrow\rangle & |\downarrow\rangle|\downarrow\rangle & -1 \\ |\downarrow\rangle & |\uparrow\rangle & |\downarrow\rangle|\uparrow\rangle & 0 \\ |\uparrow\rangle & |\downarrow\rangle & |\uparrow\rangle|\uparrow\rangle & 0 \\ |\uparrow\rangle & |\uparrow\rangle & |\uparrow\rangle|\downarrow\rangle & +1\end{array}$

Table 1: CNOT gate implemented on two spin qubits. The last column gives the projection of the total spin of two qubits to the $z$ axis. $\uparrow$ corresponds to "1" (true), and $\downarrow$ corresponds to "0" (false). The first qubit in the pair is considered as a control qubit. The value of the second qubit is changed only in case the first qubit is in "true" state $|\uparrow\rangle$; otherwise the state of the second qubit is not affected

Any two-level quantum system can be used as a quantum bit. Among many suggested implementations of quantum bits, viz. nuclear magnetic resonance [18], trapped ions [19], cavity electrodynamics [20], the usage of the quantum dot electron spin has a number of advantages: the qubit represented by a real $S U(2)$ spin is always well defined qubit without a possibility to dissipate; its decoherence time is much longer than that of other type qubits: for GaAs the spin decoherence time is of microsecond order [6, 3]. The control over the entanglement in a pair of quantum dot spin qubits can be performed by changing the coupling constant $J(t)$ in the Heisenberg Hamiltonian

$$
H_{S}=J(t) \boldsymbol{S}_{1} \cdot \boldsymbol{S}_{2}
$$

with typical switching time $\tau_{s}$ of the coupling constant $J(t)$ being of nanosecond order: the condition $\int_{0}^{\tau_{s}} J(t) d t=J_{0} \tau_{s}=\pi \bmod 2 \pi$ swaps the states of the qubits $\boldsymbol{S}_{1}$ and $\boldsymbol{S}_{2}$ [21].

To access the states of two spin qubits in the CNOT gate hierarchically, i.e. as the states of a composite boson, is to use the pair of operators $\hat{S}^{2}$ and $\hat{S}_{z}$ of the composite boson. In the polarization basis (5) the CNOT gate matrix is written in the form

$$
C=\left(\begin{array}{llll}
1 & 0 & 0 & 0 \\
0 & 1 & 0 & 0 \\
0 & 0 & 0 & 1 \\
0 & 0 & 1 & 0
\end{array}\right)
$$

Since the multiplet states $\left(S, S_{z}\right)$ are linearly expressed in terms of the polarization states (5)), see Eq.(7), the CNOT gate in the multiplet basis (6) is given by the matrix

$$
F=A^{-1} C A, \quad F=\left(\begin{array}{cccc}
\frac{1}{2} & 0 & -\frac{1}{2} & \frac{1}{\sqrt{2}} \\
0 & 1 & 0 & 0 \\
-\frac{1}{2} & 0 & \frac{1}{2} & \frac{1}{\sqrt{2}} \\
\frac{1}{\sqrt{2}} & 0 & \frac{1}{\sqrt{2}} & 0
\end{array}\right)
$$


The implementation of the CNOT gate (9) requires mixing between triplet and singlet states. This mixing is almost impossible for ordinary atoms, but can be easily performed on quantum dots subjected to oscillating electromagnetic field [22, 23, 24].

\section{Implementation on quantum dots}

To build hierarchic memory register (embedded in nanostructure) one needs an array of switching elements, the states of which are reliably controlled by external fields. Spinbased devices are promising for such applications in both conventional and quantum memory elements [25, 3, 16]. The decoherence time of a charge qubit is of nanosecond order, i.e. $10^{3}$ times shorter than that of the spin qubit [26]. On the other hand the desired number of qubits in a quantum dot array can be entangled by changing the electromagnetic field acting on the array [4]. Unlike real atoms, the singlet and triplet energy levels of GaAs quantum dots in an array can be easily controlled by changing the magnetic field and the interdot distance [27, 22, 28, 29, 30].

The quantum gates can be implemented either by changing tunneling barrier between neighboring single-electron quantum dots [21, 31, or by monitoring singlet-triplet transitions in two-electron quantum dot by means of spectroscopic manipulations [23, 24, 32]. Both ways are technologically feasible for GaAs heterostructures, where quantum dots with arbitrary number of excess electrons can be formed [33, 4].

A realization of quantum gates on the spin degrees of freedom of the coupled quantum dots have been proposed in [21, 3]. Similarly to the proposed realization of the CNOT (CROT) gate on the excitonic excitations in a pair of coupled quantum dots [34], a pair of merged quantum dots in a double-well potential, see Fig. 1, allows for a four distinct spin states (5).

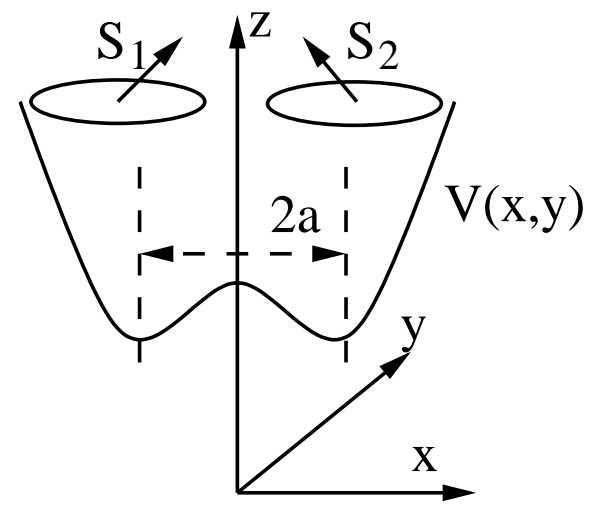

Figure 1: Two coupled one-electron quantum dots separated by the distance $2 a$ form a quantum gate. Magnetic field $B$ is applied along the $z$ direction. The harmonic wells are centered at $( \pm a, 0,0)$. The bias electric field can be applied in $x$ direction 
The Hamiltonian of coupled single-electron quantum dots has the form

$$
H=H_{\text {kinetic }}+H_{\text {potential }}+H_{\text {Zeeman }}+H_{S}
$$

where $H_{\text {kinetic }}$ is the kinetic term,

$$
H_{\text {potential }}=V(x, y)+\frac{e^{2}}{\varepsilon\left|r_{1}-r_{2}\right|}+e \sum_{i=1}^{2} x_{i} E
$$

includes the quantum dot confining potential $V(x, y)$, Coulomb repulsion of the excess electrons, and the action of the bias electric field $E$. The Zeeman splitting term is

$$
H_{\text {Zeeman }}=g \mu_{B} \sum_{i} \boldsymbol{B} \cdot \boldsymbol{S}_{i},
$$

and the Heisenberg Hamiltonian $H_{S}$ is given by (8). The confining potential is defined as

$$
V(x, y)=\frac{m \omega_{0}^{2}}{2}\left[\frac{\left(x^{2}-a^{2}\right)^{2}}{4 a^{2}}+y^{2}\right] .
$$

The typical parameters of a quantum dot in GaAs, described in [3], are:

$$
g \approx-0.44, \hbar \omega_{0}=3 \mathrm{meV}, m=0.067 m_{e}, \varepsilon=13.1 .
$$

The Bohr radius of harmonic confinement with the above listed parameters is

$$
a_{B}=\sqrt{\frac{\hbar}{m \omega_{0}}} \approx 20 \mathrm{~nm} .
$$

The value of the spin-spin coupling constant (8) for a pair of coupled single-electron quantum dots is [3]:

$$
J=\frac{\hbar \omega_{0}}{\sinh \left[2 d^{2}\left(2 b-\frac{1}{b}\right)\right]}\left[c \sqrt{b}\left\{e^{-b d^{2}} I_{0}\left(b d^{2}\right)-e^{d^{2}(b-1 / b)} I_{0}\left(d^{2}(b-1 / b)\right)\right\}+\frac{3}{4 b}\left(1+b d^{2}\right)\right],
$$

where $b=\frac{\omega}{\omega_{0}}=\sqrt{1+\left(\frac{\omega_{L}}{\omega_{0}}\right)^{2}}$ is dimensionless magnetic field, $\omega_{L}=\sqrt{\frac{e B}{2 m c}}$ is the Larmor frequency, $I_{0}$ is the zeroth-order Bessel function. Varying the magnetic field $B$ in the range $0-2 \mathrm{~T}$ one can control the value and the sign of the coupling $J$ in the range about $\pm 1 \mathrm{meV}$. The energy difference between the singlet and the triplet states in two-electron quantum dots can be found in [22].

Quantum XOR, or the CNOT, gate can be obtained by applying a sequence of operations, consisting of single qubit rotations and the swapping of two qubits [21]:

$$
U_{X O R}=e^{\imath \frac{\pi}{2} S_{1 z}} e^{-\imath \frac{\pi}{2} S_{2 z}} U_{\text {swap }}^{\frac{1}{2}} e^{\imath \pi S_{1 z}} U_{\text {swap }}^{\frac{1}{2}} .
$$


The swapping of two spin states is provided by the Heisenberg Hamiltonian (8) with the condition for pulse duration $\int_{0}^{\tau_{s}} J(t) d t=J_{0} \tau_{s}=\pi \bmod 2 \pi$ provided swapping the states of the qubits $\boldsymbol{S}_{1}$ and $\boldsymbol{S}_{2}$ by unitary operator

$$
U_{s}(t)=\mathrm{T} e^{\imath \int_{0}^{t} H_{S}(\tau) d \tau}
$$

Since the Hamiltonian (8) can be expressed in terms of the total spin $\hat{S}=\hat{S}_{1}+\hat{S}_{2}$ :

$$
H_{S}=\frac{J}{2}\left[\hat{S}^{2}-\frac{3}{2}\right],
$$

the coupling constant $J$ determines the energy difference between the singlet, and the triplet state and the swapping operation is performed by action on the spin states of the compound two electron system. The read-out of the final state can be performed either by spin-to-charge conversion for a single electron tunneling off the dot [21, 35], or by optical spectroscopy of the quantum dot state [24]. The $\sqrt{\text { swap }}$ operations have been performed experimentally on quantum dots with the operation time of 180ps [36].

The fluctuations of magnetic field do not affect the coherence of the spin states if their length is much greater than the magnetic length of quantum dot, which is of $10^{1} \mathrm{~nm}$ order. We can also neglect the spin-orbital coupling [37,

$$
H_{S O}=\frac{\omega_{0}^{2}}{2 m c^{2}} \boldsymbol{L} \cdot \boldsymbol{S}
$$

since $H_{S O} / \hbar \omega_{0} \sim 10^{-7}$. As a consequence of this, the dephasing effects caused by charge density fluctuations can significantly affect only the charge degrees of freedom, but have little effect on the spin (except for the case when the Coulomb repulsion of the electrons is significant [38]). The interaction between the spins of different quantum dots is proportional to the inverse third power of the distance. The strength of this interaction can be controlled by making the sequence of quantum dots aperiodic. The dipole interaction between the spin qubits and the surroundings spins of the environment can be estimated as $\left(g \mu_{B}\right)^{2} / a_{B}^{3} \approx 10^{-9}$ meV for GaAs quantum dots [3], which is very small. The only significant source of dephasing is the hyperfine interaction with nuclear spins, which, however, can be strongly suppressed either by dynamically polarizing the nuclear spins, or by applying magnetic field [3, 39].

\section{Conclusion}

In this paper we present an analog of the Mallat sequence for pyramidal information compression, widely implemented in classical computing as discrete wavelet transform (DWT), for the case of quantum memory on electron spins. The known quantum analogs of the DWT are based on a register of quantum bits connected by a quantum network, evaluating sums and differences of the qubit functions, same as for classical Haar wavelet algorithm (2). As 
an any scheme that addresses each qubit separately, such scheme faces the usual scalability problem of quantum computation. Our method of information compression uses the multiplet decomposition of the whole space of spin states of the memory, instead of addressing each qubit separately. Doing so, we avoid the problem of decoherence caused by the local information transmission to a given qubit, which constrains miniaturization of information processing devices and implies extra restrictions on geometric equality of memory elements.

Addressing different spin states of the whole system, rather than different quantum bits, can allow for a "flexible" memory elements (say, on aperiodic sequences of quantum dots [27]). The price paid for such flexibility is the spectroscopic problem to distinguish reliably the spin states of quantum system containing 2,4 and more $2^{M}$ spins. Since the size of the physical support of the group of spins, manipulated spectroscopically, say a group of excess electrons in quantum dots, is comparable to the size of single qubit of the same nature, our method possibly provides a new way of miniaturization of memory elements on nanoscale heterostructures.

\section{Acknowledgment}

The authors are thankful to Dr. V.N.Gorbachev for useful discussions. The research was supported in part by the RFBR Project 11-02-00604-a and the Program of Creation and Development of the National University of Science and Technology "MISiS".

\section{References}

[1] M.A. Nielsen and I.L Chuang. Quantum computation and quantum information. Cambridge University Press, NY, 2000.

[2] J. Stolze and D. Suter. Quantum computing: A short course from theory to experiment. Wiley-VCH, 2008.

[3] G. Burkard, D. Loss, and D.P. DiVincenzo. Coupled quantum dots as quantum gates. Phys. Rev. B, 59(3):2070-2078, 1999.

[4] R. Hanson, L.P. Kouwenhoven, J.R. Petta, S. Tarucha, and L. M. K. Vandersypen. Spins in few-electron quantum dots. Rev. Mod. Phys., 79(4):1217-1265, 2007.

[5] A.V. Khaetskii and Yu. V. Nazarov. Spin relaxation in semiconductor quantum dots. Phys. Rev. B, 61(19):12639-12642, 2000.

[6] J.M. Kikkawa and D.D. Awschalom. Resonant spin amplification in $n$-type GaAs. Phys. Rev. Lett., 80(19):4313-4316, 1998. 
[7] P. Burt and E. Adelson. The Laplacian pyramid as a compact image code. IEEE Trans. Comm., 31:482-540, 1983.

[8] I. Daubechies. Orthonormal bases of compactly supported wavelets. Comm. Pure. Apl. Math., 41:909-996, 1988.

[9] S. Mallat. A theory for multiresolution signal decomposition: wavelet transform. Preprint GRASP Lab. Dept. of Computer an Information Science, Univ. of Pensilvania, 1986.

[10] P. Hoyer. Efficient quantum transforms. Arxiv preprint quant-ph/9702028, 1997.

[11] A. Fijany and C. Williams. Quantum wavelet transforms: Fast algorithms and complete circuits. Lecture Notes in Computer Science, 1509:10-33, 1999.

[12] D.M. Brink and G.R. Satchler. Angular Momentum. Oxford University Press, 1994.

[13] M. V. Altaisky. Quantum states of hierarchic systems. Int. J. Quantum Information, $1(2): 269-278,2003$.

[14] K. Nomoto, R. Ugajin, T. Suzuki, and I. Hase. Single electron-photon logic device using coupled quantum dots: Computation with the fock ground state. Journal of applied physics, 79(1):291-300, 1996.

[15] A.O. Orlov, I. Amlani, G.H. Bernstein, C.S. Lent, and G.L. Snider. Realization of a functional cell for quantum-dot cellular automata. Science, 277(5328):928, 1997.

[16] P. Recher, E.V. Sukhorukov, and D. Loss. Quantum dot as spin filter and spin memory. Phys. Rev. Lett., 85(9):1962-1965, 2000.

[17] A. Barenco, D. Deutsch, A. Ekert, and R. Jozsa. Conditional quantum dynamics and logic gates. Physical Review Letters, 74(20):4083-4086, 1995.

[18] I.L. Chuang, N. Gershenfeld, and M. Kubinec. Experimental implementation of fast quantum searching. Physical review letters, 80(15):3408-3411, 1998.

[19] J.I. Cirac and P. Zoller. Quantum computations with cold trapped ions. Physical Review Letters, 74(20):4091-4094, 1995.

[20] Q.A. Turchette, CJ Hood, W. Lange, H. Mabuchi, and H.J. Kimble. Measurement of conditional phase shifts for quantum logic. Physical Review Letters, 75(25):4710-4713, 1995.

[21] D. Loss and D.P. DiVincenzo. Quantum computation with quantum dots. Phys. Rev. A, 57(1):120-126, 1998. 
[22] M Wagner, U. Merkt, and A.V. Chaplik. Spin-singlet-spint-triplet oscillations in quantum dots. Physical Review B, 45(4):1951-1954, 1992.

[23] S. Dickmann and P. Hawrylak. Spin-singlet-spin-triplet transitions in quantum dots. Journal Superconductivity: Incorporating Novel Magnetism, 16(2):387-390, 2003.

[24] D. Banerjee, P. Goswami, S. Das, and J.K. Bhattacharjee. Singlet-triplet transitions in two-electron quantum dots: Raman spectroscopic and photoelectric study. J. Phys. B: At. Mol. Opt. Phys., 41:175001, 2008.

[25] G.A. Prinz. Spin-polarized transport. Physics Today, 48(4):58, 1995.

[26] A. G. Huibers, M. Switkes, C. M. Marcus, K. Campman, and A. C. Gossard. Dephasing in open quantum dots. Phys. Rev. Lett., 81:200-203, Jul 1998.

[27] N.E. Kaputkina, Yu.E. Lozovik, R.F. Muntyanu, and Y.K. Vekilov. Aperiodic arrays of quantum dots: Influence of external magnetic and electric fields. In Journal of Physics: Conference Series, volume 226, page 012028, 2010.

[28] D. Pfannkuche, V. Gudmundsson, and P.A. Maksym. Comparison of a Hartree, a Hartree-Fock, and an exact treatment of quantum-dot Helium. Phys. Rev. B, 47(4):2244-2250, 1993.

[29] Ya. M. Blanter, N.E. Kaputkina, and Yu. E. Lozovik. Two - electron quantum dots in magnetic field. Physica Scripta, 54:539-541, 1996.

[30] Yu.E. Lozovik and N.E. Kaputkina. Quantum crystallyzation in two-electron quantum dot in magnetic field. Physica Scripta, 57:538-541, 1998.

[31] F. R. Waugh, M. J. Berry, C. H. Crouch, C. Livermore, D. J. Mar, R. M. Westervelt, K. L. Campman, and A. C. Gossard. Measuring interactions between tunnel-coupled quantum dots. Phys. Rev. B, 53:1413-1420, Jan 1996.

[32] T. Köppen, D. Franz, A. Schramm, Ch. Heyn, D. Heitmann, and T. Kipp. Resonant Raman transitions into singlet and triplet states in ingaas quantum dots containing two electrons. Phys. Rev. Lett., 103(3):037402, 2009.

[33] S. Tarucha, D. G. Austing, T. Honda, R. J. van der Hage, and L. P. Kouwenhoven. Shell filling and spin effects in a few electron quantum dot. Phys. Rev. Lett., 77:3613-3616, 1996.

[34] X. Li, Y. Wu, D. Steel, D. Gammon, T.H. Stievater, D.S. Katzer, D. Park, C. Piermarocchi, and L.J. Sham. An all-optical quantum gate in semiconductor quantum dot. Science, 301(5634):809-811, 2003. 
[35] H.-A. Engel, V. N. Golovach, D. Loss, L. M. K. Vandersypen, J. M. Elzerman, R. Hanson, and L. P. Kouwenhoven. Measurement efficiency and $n$-shot readout of spin qubits. Phys. Rev. Lett., 93:106804, Sep 2004.

[36] J.R. Petta, A.C. Johnson, J.M. Taylor, E.A. Laird, A. Yacoby, M.D. Lukin, C.M. Marcus, M.P. Hanson, and A.C. Gossard. Coherent manipulation of coupled electron spins in semiconductor quantum dots. Science, 309:2180-2184, 2005.

[37] D.P. DiVincenzo and D. Loss. Quantum information is physical. Superlattices and Microstructures, 23:419, 1998.

[38] X. Hu and S. Das Sarma. Charge-fluctuation-induced dephasing of exchange-coupled spin qubits. Phys. Rev. Lett., 96:100501, 2006.

[39] A.M. Souza, G.A. Alvarez, and D. Suter. Robust dynamical decoupling for quantum computing and quantum memory. Phys. Rev. Lett., 106:240501, 2011. 